\title{
To be or not to be
}

\section{Sean Kullman, MA}

"To be, or not to be: that is the question: Whether 'tis nobler in the mind to suffer"

The inactive verbs of the passive voice, Or to take pen against a litany of troubles,

"And by opposing end them?" Hamlet, with italics by Sean Kullman

When is it correct to use the passive voice? Avoid the passive voice when possible and use the active voice. In the active voice, the subject performs the action of the verb. In the passive voice, the subject is acted upon. For most scientists, the passive voice (verbs such as is, was, are, and were) is direct and logical. However, scientific writers need to consider alternatives to stolid language.

Incorrect: The hare was beaten by the tortoise.

Correct: The tortoise beat the hare.
(PASSIVE VOICE)

(ACTIVE VOICE)

Incorrect: A previously healthy 17 year old, $150 \mathrm{lb}$ Hispanic male presented to the Emergency Department stating that he had been bitten by a spider while sleeping.

Correct: A previously healthy 17 year old, $150 \mathrm{lb}$ Hispanic male presented to the Emergency Department after stating that a spider had bitten him.

For many writers, the passive voice is a rehearsed pattern of writing. In some instances a passive verb is necessary. In other instances passive verbs seem unclear.

Unclear: It has also been reported to be used successfully in a patient with anticholinergic drug induced ileus.

Clear: Neostigmine has been successfully used in a patient with anticholinergic drug induced ileus.

Writers can use linking verbs instead of the more stagnant forms of to be. All writers carefully choose words that best describe the actual experience in the lab or emergency room. Although scientific writing is not considered a literary genre, words that directly describe a patient's experience feel more accurate. 